\begin{tabular}{|c|l|}
\hline Title & $\begin{array}{l}\text { Preparation of platinized strontium titanate covered with hollow silica and its activity for overall water splitting in a } \\
\text { novel phase boundary photocatalytic sy stem }\end{array}$ \\
\hline Author(s) & IKEDA, Shigeru; HIRA O, Ko; ISHINO, Satoru; MA TSUMURA, Michio; OHTA NI, Bunsho \\
\hline Citation & $\begin{array}{l}\text { Catalysis Today, 117(1-3), 343 349 } \\
\text { https://doi.org/10.1016/.cattod.2006.05.037 }\end{array}$ \\
\hline Issue Date & 2006-09-30 \\
\hline Doc URL & http://hdl.handle.net/2115/48671 \\
\hline Type & article (author version) \\
\hline File Information & Catal oday117_343.pdf \\
\hline
\end{tabular}

Instructions for use 


\title{
Preparation of platinized strontium titanate covered with hollow silica and its activity for overall water splitting in a novel phase-boundary photocatalytic system
}

Shigeru IKEDA, ${ }^{\mathrm{a}, \mathrm{b}^{*}}$ Ko HIRAO, ${ }^{\mathrm{a}}$ Satoru ISHINO, ${ }^{\mathrm{a}}$ Michio MATSUMURA, ${ }^{\mathrm{a}}$ and Bunsho OHTANI ${ }^{\mathrm{c}}$

${ }^{a}$ Research Center for Solar Energy Chemistry, Osaka University, 1-3 Machikaneyama, Toyonaka, Osaka 560-8531, Japan

b“Conversion and Control by Advanced Chemistry”, PRESTO, Japan Science and Technology Agency (JST), Japan

${ }^{c}$ Catalysis Research Center, Hokkaido University, Sapporo 001-0021, Japan

\begin{abstract}
Platinum-loaded strontium titanate $\left(\mathrm{Pt}-\mathrm{SrTiO}_{3}\right)$ (core) -silica (shell) powder was prepared by double-layer winding of a carbon and a silica layer on $\mathrm{Pt}-\mathrm{SrTiO}_{3}$ followed by heat treatment to remove the carbon layer. Scanning electron microscope (SEM) observation and analyses of the BET surface area suggested that the powder has a void space between $\mathrm{Pt}-\mathrm{SrTiO}_{3}$ (core) and silica (shell). When the surface of the powder was partially modified with a fluoroalkylsilylation agent, thus-obtained material assembled at a gas-water interface and acted as a photocatalyst for overall water splitting to produce hydrogen $\left(\mathrm{H}_{2}\right)$ and oxygen $\left(\mathrm{O}_{2}\right)$. Probably due to the suppression of a backward reaction, production of water from $\mathrm{H}_{2}$ and $\mathrm{O}_{2}$, on the platinum, the overall efficiency of this system was higher than that of the conventional suspension system. Moreover, while the $\mathrm{Pt}-\mathrm{SrTiO}_{3}$ powders directly covered with fluoroalkylethylsilyl groups showed low photostability, i.e., prolonged irradiation precipitated some of the surface-modified
\end{abstract}


particles in water owing to photocatalytic decomposition of surface fluoroalkylethylsilyl groups, this material could retain its location at the phase boundary.

\section{Keywords}

photocatalytic water splitting; platinum loaded photocatalysts; gas-water interface; silica coating; amphiphilicity; core-shell structure; void space; backward reaction; photostability.

*Corresponding author.

Tel: +81-6-6850-6696; fax; +81-6-6850-6699

E-mail address: sikeda@chem.es.osaka-u.ac.jp

\section{Introduction}

Overall water splitting is one of the most exciting reactions in photocatalysis since it has potential application to direct production of $\mathrm{H}_{2}$ for clean energy using photon energy $[1,2]$. A considerable number of solid photocatalysts for overall water splitting have been discovered in extensive research [3-12]. In most cases, photocatalysts utilized for the reaction are formed by the combination of transition metal oxides and promoters: the former has the function of generating photoexcited electrons and holes, whereas the latter, usually fine metal or metal oxide particles dispersed on metal oxides, acts as a reduction catalyst of water into $\mathrm{H}_{2}$.

Among reduction catalysts, platinum $(\mathrm{Pt})$ is the best metal for $\mathrm{H}_{2}$ liberation because of its smallest overpotential of water reduction. Indeed, many studies have shown that Pt-loaded photocatalysts show efficient activity for $\mathrm{H}_{2}$ liberation from 
aqueous suspensions containing sacrificial reagents such as alcohols [13-16]. However, the overall water splitting reaction, stoichiometric photocatalytic decomposition of water into $\mathrm{H}_{2}$ and $\mathrm{O}_{2}$, scarcely proceeds on Pt-loaded photocatalysts under ambient conditions because of the high activity of $\mathrm{Pt}$ for a backward reaction, i.e., production of water from $\mathrm{H}_{2}$ and $\mathrm{O}_{2}$.

In the past decade, some researchers have succeeded in overcoming the problem of photocatalytic overall water splitting on Pt-loaded photocatalysts by improving reaction conditions and photocatalysts [17-19]. Sato et al. showed in their pioneering work on Pt-loaded photocatalytic systems that Pt-loaded $\mathrm{TiO}_{2}\left(\mathrm{Pt}-\mathrm{TiO}_{2}\right)$ coated with $\mathrm{NaOH}$ could decompose water vapor [17]. Tabata et al. reported that the overall water splitting of pure water can be achieved by just changing the mode of photoirradiation; an aqueous $\mathrm{Pt}-\mathrm{TiO}_{2}$ suspension was irradiated from the top of a reaction cell but not from the bottom or side of the cell [18]. They claimed that the further the photoirradiated particles are from the water level, the faster is the backward reaction of $\mathrm{H}_{2}$ and $\mathrm{O}_{2}$ suppressing the overall rate, and top-irradiation reduces the distance, resulting in higher efficiency. From their findings, the appropriate condition for overall water splitting on Pt-loaded photocatalysts seems to be that photocatalysts presented at the gas-water interface.

Recently, we have found a facile method for assembling oxide materials at oil-water and gas-water interfaces by partial modification of external surfaces of particles with an alkylsilane agent [20-25]. One of the most noticeable features of these amphiphilic materials is that the assembled particles loaded with small amounts of titanium(IV) oxide acted as an efficient catalyst for oxidation of several hydrophobic alkenes and alcohols with aqueous hydrogen peroxide without mechanical agitation [20-23]. The reaction system is thus named phase-boundary catalysis.

We have also proposed the possibility of application of a phase-boundary catalytic system to liquid-liquid and gas-liquid dual-phase photocatalytic reactions [26]. For 
these systems, it was confirmed that alkylsilane agents should be loaded indirectly to the surface of photocatalysts in order to inhibit photocatalytic decomposition of surface-covered alkylsilyl groups: the photostability was considerably improved when commercially available $\mathrm{TiO}_{2}$ particles coated with porous silica $\left(\mathrm{SiO}_{2}\right)$ [27] were employed as a starting material and the surface of $\mathrm{SiO}_{2}$ was modified with an alkylsilane agent.

On the basis of these facts and findings, our research interest is focused on development of a system for efficient photocatalytic overall splitting using Pt-loaded photocatalysts assembled at the gas-water interface. In this study, we prepared Pt-loaded $\mathrm{SrTiO}_{3}$ photocatalysts covered with hollow silica, made their surfaces amphiphilic so that the particles would assemble at the gas-water interface, and used them for the phase-boundary photocatalytic water splitting.

\section{Experimental}

\subsection{Preparation of Pt-loaded $\mathrm{SrTiO}_{3}$}

Platinum-loaded $\mathrm{SrTiO}_{3}\left(\mathrm{Pt}-\mathrm{SrTiO}_{3}\right)$ was prepared by a photodeposition method [15]. Typically, $1 \mathrm{~g}$ of $\mathrm{SrTiO}_{3}$ (Fuji Titanium Industry) suspended in an aqueous solution $\left(150 \mathrm{~cm}^{3}\right)$ containing methanol $(10 \mathrm{vol} \%)$ and $5 \mu \mathrm{mol}$ of chloroplatinic acid $\left(\mathrm{H}_{2} \mathrm{PtCl}_{6}\right.$ $6 \mathrm{H}_{2} \mathrm{O}$ ) was photoirradiated (> $290 \mathrm{~nm}$, see below) in argon $(\mathrm{Ar})$ for $3 \mathrm{~h}$ with magnetic stirring (ca. $1000 \mathrm{rpm}$ ) and this was followed by washing with water several times and drying to achieve $0.1 \mathrm{wt} \%$ loading of Pt.

\subsection{Incorporation of Pt-SrTiO ${ }_{3}$ into hollow silica}


Incorporation of $\mathrm{Pt}-\mathrm{SrTiO}_{3}$ into hollow silica was conducted by successive coating of $\mathrm{Pt}-\mathrm{SrTiO}_{3}$ with a carbon layer and a silica layer followed by heat treatment to remove the carbon layer. First, a carbon layer was coated on $\mathrm{Pt}-\mathrm{SrTiO}_{3}$ by using a procedure similar to that used for carbon-coatings of silver and gold nanoparticles [28]. To an aqueous solution $\left(80 \mathrm{~cm}^{3}\right)$ containing $40 \mathrm{mmol}$ of glucose, $0.5 \mathrm{~g}$ of $\mathrm{Pt}_{-} \mathrm{SrTiO}_{3}$ was added. The suspension was placed in a Teflon-sealed autoclave and maintained at $453 \mathrm{~K}$ for $3 \mathrm{~h}$. The brown powder was isolated by filtration, washed with water and ethanol, and heated at $773 \mathrm{~K}$ under evacuation for $2 \mathrm{~h}$. Then $0.3 \mathrm{~g}$ of carbon-coated $\mathrm{Pt}_{-} \mathrm{SrTiO}_{3}$ $\left(\mathrm{c} / \mathrm{Pt}-\mathrm{SrTiO}_{3}\right)$ thus-obtained was suspended in a mixed solution of ethanol $\left(60 \mathrm{~cm}^{3}\right)$ and water $\left(5 \mathrm{~cm}^{3}\right)$ containing $150 \mu \mathrm{mol}$ of aminopropyltriethoxysilane (APS). After stirring the suspension for $24 \mathrm{~h}$ at room temperature, $500 \mu \mathrm{mol}$ of tetraethyl orthosilicate (TEOS) was added and the suspension was further stirred for $24 \mathrm{~h}$. The solid part was collected by filtration, washed with water several times, and heated at $783 \mathrm{~K}$ under vacuum to yield silica-coated c/Pt-SrTiO 3 ( $\left.\mathrm{si} / \mathrm{c} / \mathrm{Pt}-\mathrm{SrTiO}_{3}\right)$. Finally, the carbon component in the si/c/Pt-SrTiO 3 powder was removed by heating the sample at $873 \mathrm{~K}$ for $3 \mathrm{~h}$ in air to obtain $\mathrm{Pt}-\mathrm{SrTiO}_{3}$ incorporated into the silica layer $\left(p-\mathrm{si} / \mathrm{Pt}-\mathrm{SrTiO}_{3}\right)$. As a reference, $\mathrm{Pt}-\mathrm{SrTiO}_{3}$ coated with silica $\left(n-\mathrm{si} / \mathrm{Pt}-\mathrm{SrTiO}_{3}\right)$ was prepared without coating with carbon in the first step of the procedure for preparing $p$-si/Pt-SrTiO ${ }_{3}$.

\subsection{Modification of particle surface with a fluoroalkylethlysilylation agent}

Functionalization of external surfaces of $\mathrm{Pt}-\mathrm{SrTiO}_{3}$, and $p$-si $/ \mathrm{Pt}-\mathrm{SrTiO}_{3}$ with fluoroalkylethylsilyl (FES) groups was carried out by following the procedure for partial modification of zeolite, silica, and titanium(IV) oxide $\left(\mathrm{TiO}_{2}\right)$ with alkylsilyl groups [20-26]. A schematic illustration of the procedure is shown in Scheme 1. To 0.2 $\mathrm{g}$ of $\mathrm{Pt}-\mathrm{SrTiO}_{3}$ or $p$-si/Pt-SrTiO $3,0.03 \mathrm{~cm}^{3}$ of water was added and the mixture was stirred until the added water had been soaked into the particles. Then thus-obtained 
Pt-SrTiO 3 or $p$-si $/ \mathrm{Pt}_{-} \mathrm{SrTiO}_{3}$ aggregates were suspended in $3 \mathrm{~g}$ of toluene containing 20 $\mu$ mol of tridecafluoro-1.1.2.2.-tetrahydrooctyltrichlorosilane (TDFS). After shaking the mixture for $5 \mathrm{~min}$ at room temperature, the suspension was centrifuged and washed with toluene (ca. $5 \mathrm{~g}$ ) and ethanol (ca. $5 \mathrm{~g}$ ) to remove unreacted TDFS. The precipitate was then dried at $383 \mathrm{~K}$ overnight under evacuation. The resulting powders were labeled w/o-Pt/SrTiO 3 and w/o-p-si/Pt-SrTiO ${ }_{3}$, respectively. For comparison, a Pt-SrTiO powder fully covered with FES groups (o-Pt-SrTiO ${ }_{3}$ ) was prepared without mixing water in the first step of the above-described procedure for preparation of w/o-samples.

\subsection{Characterization and analytical procedures}

SEM images were taken using a Hitachi S-5000 FEG scanning electron microscope at a voltage of $20 \mathrm{kV}$. Thermogravimetry-differential thermal analysis (TG-DTA) was conducted using a Bruker 2000A TG-DTA apparatus in air (flow rate:

$50 \mathrm{~cm}^{3} \mathrm{~min}^{-1}$ ) from room temperature to $1173 \mathrm{~K}$, with a heating ramp of $10 \mathrm{~K} \mathrm{~min}^{-1}$. Amounts of FES groups and silica on the surface of $\mathrm{Pt}_{-} \mathrm{SrTiO}_{3}$ were determined by using a Nippon Jarrel-Ash ICPAP-575 Mark II inductively coupled plasma atomic emission spectrometer (ICP-AES). For the measurement, siliceous components on the samples were dissolved by dispersing the samples in a $0.05 \mathrm{~mol} \mathrm{dm}^{3} \mathrm{NaOH}$ solution for $6 \mathrm{~h}$ at $333 \mathrm{~K}$, and the solution part was collected by centrifugal removal of remaining solid parts. Samples containing FES groups were calcined at $873 \mathrm{~K}$ to convert the FES groups into $\mathrm{SiO}_{2}$ before the treatment with $\mathrm{NaOH}$ solution.

\subsection{Photoirradiation and product analysis}

The photocatalytic runs for water splitting reaction were performed in an air-free closed gas circulation system with a Pyrex reaction cell. Each sample $(50 \mathrm{mg})$ was 
added to $150 \mathrm{~cm}^{3}$ of water and phoroirradiated in Ar. Irradiation with light of wavelength from a ultrahigh-pressure mercury arc (Ushio, $1 \mathrm{~kW}$ ) was performed through the top of a Pyrex glass reaction vessel, so that light of wavelength longer than $290 \mathrm{~nm}$ reached the suspension. The temperatures of suspensions under photoirradiation were kept at $298 \mathrm{~K}$ in a thermostatted water bath. The amounts of $\mathrm{H}_{2}$ and $\mathrm{O}_{2}$ evolved were determined using a Shimadzu GC-8A gas chromatograph equipped with an MS-5A column (GL Sciences) and a TCD detector through a gas sampler $\left(3 \mathrm{~cm}^{3}\right)$ that was directly connected to the reaction system to prevent any contamination from air.

The apparent quantum efficiency, defined as molar ratio of the product (twice the molar amount of $\mathrm{H}_{2}$ ) to the incident photons, was roughly estimated using the flux of incident photons of wavelength between 290 and $390 \mathrm{~nm}$ by assuming that all the photons of energy greater than their band gap $(3.2 \mathrm{eV})$ were absorbed by present Pt-SrTiO 3 -based photocatalysts. The incident photon flux was evaluated by total emission energy of the mercury arc at the sample position and a spectrum measured using an Ushio USR-40V/D spectra radiometer.

\section{Results and Discussion}

\subsection{Structural characterization of $\mathrm{Pt}-\mathrm{SrTiO}_{3}$ covered with hollow silica}

Figure 1 shows SEM images of $\mathrm{Pt}-\mathrm{SrTiO}_{3}, \mathrm{c} / \mathrm{SrTiO}_{3}, \mathrm{si} / \mathrm{c} / \mathrm{SrTiO}_{3}$, and $p$-si $/ \mathrm{SrTiO}_{3}$. The Pt-SrTiO ${ }_{3}$ samples exhibited angular morphology reflecting a cubic perovskite structure. Carbonization of glucose in the presence of $\mathrm{Pt}_{-} \mathrm{SrTiO}_{3}$ produced a carbon layer on the surface of $\mathrm{Pt}-\mathrm{SrTiO}_{3}$ : a carbon layer of ca. $20 \mathrm{~nm}$ in thickness entirely covers the Pt-SrTiO 3 powders (Fig. 1b). The smooth surface of c/ $\mathrm{Pt}_{-} \mathrm{SrTiO}_{3}$ altered slightly to rough state after treatment of c/Pt-SrTiO 3 with APTS and TEOS (Fig. 1c). 
After heat treatment of si/c/Pt-SrTiO 3 at $873 \mathrm{~K}$ in air, on the other hand, there was no significant change in morphology, i.e., the presence of a thin layer surrounding Pr-SrTiO 3 particles was clearly observed (Fig. 1d). From the fact that no apparent weight loss accompanied by an exothermic event was observed by TG-DTA analysis of the $p$-si/Pt-SrTiO ${ }_{3}$ sample, complete removal of carbon components should be achieved during the heat treatment of si/c/Pt-SrTiO 3 . Moreover, ICP-AES analysis for silicon indicated the presence of $2.3 \mathrm{wt} \%$ of silicon, corresponding to ca. $5 \mathrm{wt} \%$ of $\mathrm{SiO}_{2}$, on $p$-si/Pt-SrTiO ${ }_{3}$. These results indicate that the surface-covered layer on the p-si/Pt-SrTiO 3 sample consists of $\mathrm{SiO}_{2}$, i.e., the $p$-si/ $\mathrm{Pt}-\mathrm{SrTiO}_{3}$ particle has a core $\left(\mathrm{Pt}-\mathrm{SrTiO}_{3}\right)-$ shell $\left(\mathrm{SiO}_{2}\right)$ structure. Another notable feature shown in the SEM images of $p$-si $/ \mathrm{SrTiO}_{3}$ is that there seems to be a void space between the core $\left(\mathrm{SrTiO}_{3}\right)$ and shell $\left(\mathrm{SiO}_{2}\right)$ where the carbon layer originally existed. The presence of a void space in $p$-si/Pt-SrTiO 3 is also supported by its relatively high BET surface area $\left(20 \mathrm{~m}^{2} \mathrm{~g}^{-1}\right)$ in comparison with that of the original $\mathrm{Pt}-\mathrm{SrTiO}_{3}$ sample $\left(4 \mathrm{~m}^{2} \mathrm{~g}^{-1}\right)$.

\subsection{Surface characteristics of particles covered with FES groups}

In order to assemble the $\mathrm{Pt}-\mathrm{SrTiO}_{3}$ and $p$-si/Pt-SrTiO 3 powders at the gas-water interface, it is necessary to give the particles hydrophobicity because these powders are originally hydrophilic owing to their surface hydroxyl groups. Moreover, the assembled particles should have affinity with water to induce the overall water splitting efficiently (see below). Thus, we prepared amphiphilic $\mathrm{Pt}_{-} \mathrm{SrTiO}_{3}$ and $p$-si/Pt-SrTiO ${ }_{3}$ powders (w/o-Pt-SrTiO 3 and w/o-p-si/Pt-SrTiO ${ }_{3}$, respectively) by the procedure shown in Scheme 1. The strategy to make amphiphilic powders is based on aggregation of the originally hydrophilic particles by addition of a small amount of water just before the reaction with TDFS. Since the binding water between particles prevents penetration of the agent into the aggregates, FES groups can attach only on the outer parts of 
aggregates. Therefore, the surfaces of the resulting powders were amphiphilic, having both hydroxyl and FES faces.

Figure 2 shows TG curves of the original $\mathrm{Pt}-\mathrm{SrTiO}_{3}$ and $\mathrm{Pt}-\mathrm{SrTiO}_{3}$ modified with FES groups measured in air in the temperature range of r.t.-1173 K. For all samples, a slight weight loss below $400 \mathrm{~K}$ appeared in the TG curves, probably due to dehydration and desorption of water and/or organic solvents. Moreover, samples modified with FES groups showed an additional large weight loss between ca. $500 \mathrm{~K}$ and ca. $800 \mathrm{~K}$. Since the weight loss was accompanied by a major exothermic event starting at ca. $500 \mathrm{~K}$ in DTA curves of both samples (data not shown), this was ascribed to combustion of surface-grafted FES groups on w/o-Pt-SrTiO ${ }_{3}$ and o-Pt-SrO${ }_{3}$. The observation of relatively large weight loss of o-Pt-SrO 3 in comparison with that of w/o-Pt-SrTiO suggests relatively low surface coverage of FES groups on w/o-Pt-SrTiO ${ }_{3}$.

For quantitative analysis of surface-loaded FES groups, amounts of FES groups on w/o-Pt-SrTiO 3 and o-Pt-SrTiO 3 were estimated by using ICP-AES analysis of silicon components. As a result, the amount of FES groups on w/o-Pt-SrTiO ${ }_{3}$ and that on o-Pt-SrTiO ${ }_{3}$ were determined to be 19 and $30 \mu \mathrm{mol}$ per unit weight of the sample, respectively. From the reported cross section of an FES group $\left(0.2 \mathrm{~nm}^{2}\right)$ [29] and BET surface area of $\mathrm{Pt}_{-} \mathrm{SrTiO}_{3}\left(4 \mathrm{~m}^{2} \mathrm{~g}^{-1}\right)$, we calculated the surface coverage of w/o-Pt-SrTiO 3 to be ca. $60 \%$ and that of o-Pt-SrTiO 3 to be ca. $90 \%$ when monolayer coverage of FES groups was presumed. These results support the TG-DTA results described above. Thus, as has been demonstrated using monodispersed silica particles and an alkylsilylation agent [24,25], the w/o-Pt-SrTiO 3 sample prepared by the above-described procedure is likely to have an amphiphilic structure, almost half of the surface being covered with FES groups and the remainder retaining the bare hydrophilic surface. Owing to the presence of the silica shell, the amount of FES groups in the w/o-p-si/Pt-SrTiO 3 sample could not be determined. However, from the fact that almost similar weight loss originating in the combustion of FES groups was observed on the 
TG-DTA measurement, this sample might also retain a hydrophilic region on the outside of the $\mathrm{SiO}_{2}$ shell (Scheme 1).

Figure 3 shows the apparent locations of $\mathrm{Pt}-\mathrm{SrTiO}_{3}$ and w/o-Pt-SrTiO${ }_{3}$ added to water. Pictures of w/o-Pt-SrTiO 3 and w/o-p-si/Pt-SrTiO 3 particles after photocatalytic reaction are also shown and these are discussed below. Due to the surface hydrophilicity, the $\mathrm{Pt}-\mathrm{SrTiO}_{3}$ powder was preferentially dispersed in water. On the other hand, w/o-Pt-SrTiO 3 was assembled at the gas-water phase boundary, as expected. Similar behaviors were also observed when w/o-p-si/Pt-SrTiO 3 was used instead of w/o-Pt- $\mathrm{SrTiO}_{3}$. These results and observations imply that w/o-Pt-SrTiO 3 and w/o-p-si/Pt-SrTiO 3 are assembled at the gas-water interface by facing their hydrophobic side to the gas phase and their hydrophilic side to the water phase.

3.3 Photocatalytic activity for overall water splitting on $\mathrm{Pt}_{-} \mathrm{SrTiO}_{3}$ incorporated with hollow silica particles

Table 1 summarizes initial rates of $\mathrm{H}_{2}$ and $\mathrm{O}_{2}$ liberation on unmodified and modified $\mathrm{Pt}-\mathrm{SrTiO}_{3}$ powders suspended in water. Apparent quantum yields of the reaction are also shown in this Table. Similar to the results for the $\mathrm{Pt}-\mathrm{TiO}_{2}$ photocatalyst $[18,19]$, stoichiometric liberation of $\mathrm{H}_{2}$ and $\mathrm{O}_{2}$ was observed when the suspension containing the original $\mathrm{Pt}-\mathrm{SrTiO}_{3}$ powder was irradiated from the top of the reaction cell (entry 1). On the other hand, the surface coverage with the carbon layer completely inhibited the $\mathrm{H}_{2}$ and $\mathrm{O}_{2}$ liberation: almost no $\mathrm{H}_{2}$ and $\mathrm{O}_{2}$ liberation was observed on c/Pt-SrTiO 3 and c/si/Pt-SrTiO 3 (entries 2 and 3). Moreover, when the $n$-si/Pt-SrTiO 3 powder, the $\mathrm{Pt}-\mathrm{SrTiO}_{3}$ sample directly deposited an $\mathrm{SiO}_{2}$ layer (ca. 1wt\%) on the surface, was employed, photocatalytic activity was largely suppressed (entry 4). It is likely that active sites for $\mathrm{H}_{2}$ and $\mathrm{O}_{2}$ production were covered with such surface components, resulting in prevention of the adsorption of water molecules on these sites. 
On the other hand, despite the presence of a relatively large amount of $\mathrm{SiO}_{2}$ on the surface in comparison with that on $n$-si/Pt-SrTiO 3 , $p$-si $/ \mathrm{Pt}_{-}-\mathrm{SrTiO}_{3}$ showed stoichiometric $\mathrm{H}_{2}$ and $\mathrm{O}_{2}$ liberation (entry 5). It is notable that the rate over the catalyst was comparable to that on the original $\mathrm{Pt}-\mathrm{SrTiO}_{3}$. This unusual photocatalytic activity is attributable to the presence of the void space, which leads to efficient contact between active sites on the $\mathrm{Pt}-\mathrm{SrTiO}_{3}$ surface and water.

\subsection{Phase boundary photocatalytic water splitting}

As depicted in Table 1, when the w/o-Pt-SrTiO ${ }_{3}$ powder was floated and photoirradiated, the rates of $\mathrm{H}_{2}$ and $\mathrm{O}_{2}$ liberation became faster than those obtained on the $\mathrm{Pt}-\mathrm{SrTiO}_{3}$ suspension (entry 6). This result is consistent with our expectation: the backward reaction will be suppressed when the photocatalyst particles are presented at the gas-water interface. On the other hand, the o-Pt-SrTiO ${ }_{3}$ sample, which was also floated on water, i.e., visual observation showed practically no difference from w/o-Pt-SrTiO 3 , did not induce the photocatalytic water splitting efficiently (entry 7). These results indicate that the photocatalytic reaction efficiently occurred at the hydrophilic surface of w/o-Pt-SrTiO 3 facing the aqueous phase, i.e., efficient contact of the surface of photocatalyst with water is indispensable to induce the reaction. We have reported similar effects of hydrophilic surface on photocatalytic $\mathrm{H}_{2}$ liberation from aqueous methanol solution over an amphiphilic $\mathrm{TiO}_{2}$ photocatalyst coated with silica fine particles [26].

Figure 4 shows representative time course curves of liberation of $\mathrm{H}_{2}$ and $\mathrm{O}_{2}$ on w/o-Pt- $\mathrm{SrTiO}_{3}$ floated on water. The gas phase was evacuated at 3-h intervals in this experiment. Although stoichiometric liberations of $\mathrm{H}_{2}$ and $\mathrm{O}_{2}$ with relatively fast rates were observed at the initial stage as mentioned above, the overall rate gradually decreased accompanied by sinking of the w/o-Pt-SrTiO 3 into water, as shown in Fig. 2c. 
Since the decrease in weight loss due to combustion of surface-loaded FES groups in TG-DTA was halved on the w/o-Pt-SrTiO 3 sample after 9-h photoirradiation (data not shown), the instability was attributed to photodecomposition of FES groups on the surface through the photocatalytic reaction of $\mathrm{Pt}_{-} \mathrm{SrTiO}_{3}[26,30]$.

In order to overcome the critical disadvantage of the present system, we thought that FES groups should be loaded indirectly on the surface of $\mathrm{Pt}-\mathrm{SrTiO}_{3}$. Thus, we employed $p$-si/Pt-SrTiO 3 particles and modified their surfaces with FES groups (w/o-p-si/Pt-SrTiO 3 ) to be assembled at the gas-water interface (Scheme 1). Figure 5 shows a typical result of photocatalytic water splitting on the w/o-p-si/Pt-SrTiO 3 photocatalyst. Upon photoirradiation, this photocatalyst showed constant $\mathrm{H}_{2}$ and $\mathrm{O}_{2}$ production for prolonged photoirradiation: the initial rates of $\mathrm{H}_{2}$ and $\mathrm{O}_{2}$ liberation in each run were almost constant. Moreover, the location of the particles did not change after 9-h irradiation (Fig. 2d), i.e., almost all particles were assembled at the phase boundary. Although a decrease of ca. $10 \%$ in FES groups was observed in TG-DTA probably due to the photoinduced removal of FES groups attached directly to the Pt-SrTiO 3 surface (data not shown), we confirmed that the photodecomposition was largely suppressed by the use of $p$-si/Pt-SrTiO ${ }_{3}$.

\section{Conclusions}

We have developed an integrated photocatalytic system for overall water splitting using Pt-loaded photocatalytic materials. At present, the efficiency of photocatalytic reaction $(0.0023$, see Table 1 , entry 8$)$ is low high and the reaction only occurred under ultraviolet-light irradiation because we employed commercially available $\mathrm{SrTiO}_{3}$ powder as a bare sample. However, this system can be applied to various kinds of photocatalytic materials having high activity or having response to visible light. Studies 
in these directions are now in progress.

\section{Acknowledgements}

The authors are grateful to Fuji Titanium Industry for supplying the $\mathrm{SrTiO}_{3}$ sample. Prof. T. Hirai and Prof. Y. Shiraishi (Osaka University) are acknowledged for their help in ICP-AES measurements. This research was partially supported by a Grant-in-Aid for Young Scientists (A) (No.16685020) from the Ministry of Education, Science, Sports and Culture.

\section{References}

[1] H. Gerischer, J. Electroanal. Chem. 82 (1977) 133.

[2] M. Grätzel, Energy Resources through, Photochemistry and Catalysis, Academic Press, New York, 1983.

[3] J. M. Lehn, J. P. Sauvage, R. Ziessel, Nouv. J. Chem. 4 (1980) 623.

[4] A. Kudo, K. Sayama, A. Tanaka, K. Asakura, K. Domen, K. Maruya, T. Onishi, J. Catal. 120 (1989) 120.

[5] H. G. Kim, D. W. Hwang, J. Kim, Y. G. Kim, J. S. Lee, Chem. Commun. (1999) 1077.

[6] A. Kudo, H. Kato, S. Nakagawa, J. Phys. Chem. B 104 (2000) 571.

[7] K. Domen, J. N. Kondo, M. Hara, T. Takata, Bull. Chem. Soc. Jpn. 73 (2000) 1307.

[8] A. Kudo, J. Ceram. Soc. Jpn. 109 (2001) S81.

[9] J. Sato, N. Saito, H. Nishiyama, Y. Inoue, J. Phys. Chem. B 107 (2003) 7965. 
[10] M. Machida, K. Miyazaki, S. Matsushima, M. Arai, J. Mater. Chem. 13 (2003) 1433.

[11] R. Abe, M. Higashi, Z. Zou, K. Sayama, Y. Abe, H. Arakawa, J. Phys. Chem. B $108(2004) 811$.

[12] J. Sato, N. Saito, Y. Yamada, K. Maeda, T. Takata, J. N. Kondo, M. Hara, H. Kobayashi, K. Domen, Y. Inoue, J. Am. Chem. Soc. 127 (2005) 4150.

[13] P. Pichat, J. M. Herrmann, J. Disdier, H. Courbon, M. N. Mossanega, Nouv. J. Chim. 5 (1981) 627.

[14] S.-i. Nishimoto, B. Ohtani, T. Kagiya, J. Chem. Soc., Faraday Trans. 181 (1985) 2467.

[15] S. Ikeda, N. Sugiyama, B. Pal, G. Marcí, L. Palmisano, H. Noguchi, K. Uosaki, B. Ohtani, Phys. Chem. Chem. Phys. 3 (2001) 267.

[16] R. Konta, T. Ishii, H. Kato, K. Kudo, J. Phys. Chem. B 108 (2004) 8992.

[17] S. Sato, J, M. White, Chem. Phys. Lett. 72 (1980) 83.

[18] S. Tabata, H. Nishida, Y. Masaki, K. Tabata, Catal. Lett. (1995) 34.

[19] H. Kominami, S.-y. Murakami, M. Kohno, Y. Kera, K. Okada, B. Ohtani, Phys. Chem. Chem. Phys. 3 (2001) 4102.

[20] H. Nur, S. Ikeda, B. Ohtani, Chem. Commum. (2000) 2235.

[21] H. Nur, S. Ikeda, B, Ohtani, J. Catal. 204 (2001) 402.

[22] S. Ikeda, H. Nur, T. Sawadaishi, K. Ijiro, M. Shimomura, B. Ohtani, Langmuir 17 (2001) 7976.

[23] K.-m. Choi, S. Ikeda, S. Ishino, K. Ikeue, M. Matsumura, B. Ohtani, Appl. Catal. A $278(2005) 269$.

[24] Y. K. Takahara, S. Ikeda, K. Tachi, S. Ishino, K. Ikeue, T. Sakata, T. Hasegawa, H. Mori, M. Matsumura, B. Ohtani, J. Am. Chem. Soc, 127 (2005) 6271.

[25] S. Ikeda, Y. K. Takahara, S. Ishino, T. Sakata, T. Hasegawa, H. Mori, M. Matsumura, B. Ohtani, Chem. Lett., 34 (2005) 1386. 
[26] S. Ikeda, Y. Kowata, K. Ikeue, M. Matsumura, B. Ohtani, Appl. Catal. A 265 (2004) 69.

[27] Japanese Patent 286728 (2001).

[28] X. Sun, Y. Li, Angew. Chem. Int. Ed. 43 (2004) 597.

[29] A. Fadeev, R. Helmy, S. Marcinko, Langmuir 18 (2002) 7521.

[30] T. Kato, A. Fujishima, E. Maekawa, K. Honda, Nihon Kagaku Kaishi 1 (1986) 8.

\section{Scheme and Figure Captions}

Scheme 1. Schematic representation of the procedure for the partial loading of FES groups on $\mathrm{Pt}-\mathrm{SrTiO}_{3}$ and $p$-si/Pt-SrTiO 3 .

Fig. 1. SEM images of (a) $\mathrm{Pt}_{-} \mathrm{SrTiO}_{3}$, (b) c/Pt-SrTiO ${ }_{3}$, (c) si/c/Pt-SrTiO p-si/Pt-SrTiO 3 powders. Scale bars correspond to $300 \mathrm{~nm}$.

Fig. 2. TG curves of (a) $\mathrm{Pt}_{-} \mathrm{SrTiO}_{3}$, (b) w/o-Pt-SrTiO 3 , and (c) o-Pt-SrTiO .

Fig. 3. Locations of the original Pt-SrTiO 3 , w/o-Pt-SrTiO ${ }_{3}$, w/o-p-si/Pt-SrTiO 3 added to water: (a) Pt-SrTiO 3 , (b) w/o-Pt-SrTiO 3 before photoirradiation, (c) w/o-Pt-SrTiO ${ }_{3}$, (d) w/o-p-si/Pt-SrTiO 3 after 9-h photoirradiation.

Fig. 4. Time course curves of $\mathrm{H}_{2}$ (open circles) and $\mathrm{O}_{2}$ (filled circles) liberation on w/o-Pt-SrTiO ${ }_{3}$. The reaction system was evacuated at 3-h intervals.

Fig. 5. Plots of $\mathrm{H}_{2}$ (open circles) and $\mathrm{O}_{2}$ (filled circles) liberation on w/o-p-si/Pt-SrTiO versus photoirradiation time. The reaction system was evacuated at 3-h intervals. 


\section{Table 1.}

Photocatalytic activity of unmodified and modified $\mathrm{Pt}-\mathrm{SrTiO}_{3}$ for water splitting ${ }^{\mathrm{a}}$

\begin{tabular}{|c|c|c|c|c|c|}
\hline \multirow{2}{*}{ Entry } & \multirow{2}{*}{$\mathrm{SrTiO}_{3}$} & \multirow{2}{*}{$\begin{array}{l}\mathrm{SiO}_{2}{ }^{\mathrm{b}} \\
(\mathrm{wt} \%)\end{array}$} & \multicolumn{2}{|c|}{$\operatorname{Rate}^{\mathrm{c}}\left(\mu \mathrm{mol} \mathrm{h}{ }^{-1}\right)$} & \multirow{2}{*}{$\begin{array}{l}\text { Q.E. }^{d} \\
\left(\times 10^{-3}\right)\end{array}$} \\
\hline & & & $\mathrm{H}_{2}$ & $\mathrm{O}_{2}$ & \\
\hline 1 & $\mathrm{Pt}-\mathrm{SrTiO}_{3}$ & 0 & 12.4 & 6.3 & 1.0 \\
\hline 2 & $\mathrm{c} / \mathrm{Pt}-\mathrm{SrTiO}_{3}$ & $-{ }^{e}$ & 0 & 0 & 0 \\
\hline 3 & $\mathrm{si} / \mathrm{c} / \mathrm{Pt}-\mathrm{SrTiO}_{3}$ & $\mathrm{e}^{\mathrm{e}}$ & 0 & 0 & 0 \\
\hline 4 & $n$-si/Pt-SrTiO & 1.0 & 2.7 & 0.8 & 0.2 \\
\hline 5 & $p$-si/Pt-SrTiO 3 & 4.9 & 16.1 & 7.8 & 1.2 \\
\hline 6 & w/o-Pt-SrTiO & $-{ }^{e}$ & 17.9 & 9.2 & 1.4 \\
\hline 7 & o-Pt-SrTiO & $-{ }^{e}$ & 4.1 & 1.6 & 0.3 \\
\hline 8 & w/o-p-si/Pt-SrTiO 3 & $-{ }^{e}$ & 28.7 & 13.6 & 2.3 \\
\hline
\end{tabular}

${ }^{\text {a }}$ Catalyst $(50 \mathrm{mg})$ and pure water $\left(150 \mathrm{~cm}^{3}\right)$ were added to a reaction cell and were photoirradiated from the top part of the cell under $\mathrm{Ar}(5 \mathrm{kPa})$.

${ }^{\mathrm{b}}$ Content of $\mathrm{SiO}_{2}$ on the sample determined by ICP-AES.

${ }^{\mathrm{c}}$ Initial rate for $1 \mathrm{~h}$.

${ }^{d}$ Apparent quantum efficiency estimated by the initial rate of $\mathrm{H}_{2}$ liberation and the flux of incident photons of wavelength between 290 and $390 \mathrm{~nm}$.

${ }^{\mathrm{e}}$ Not measured. 

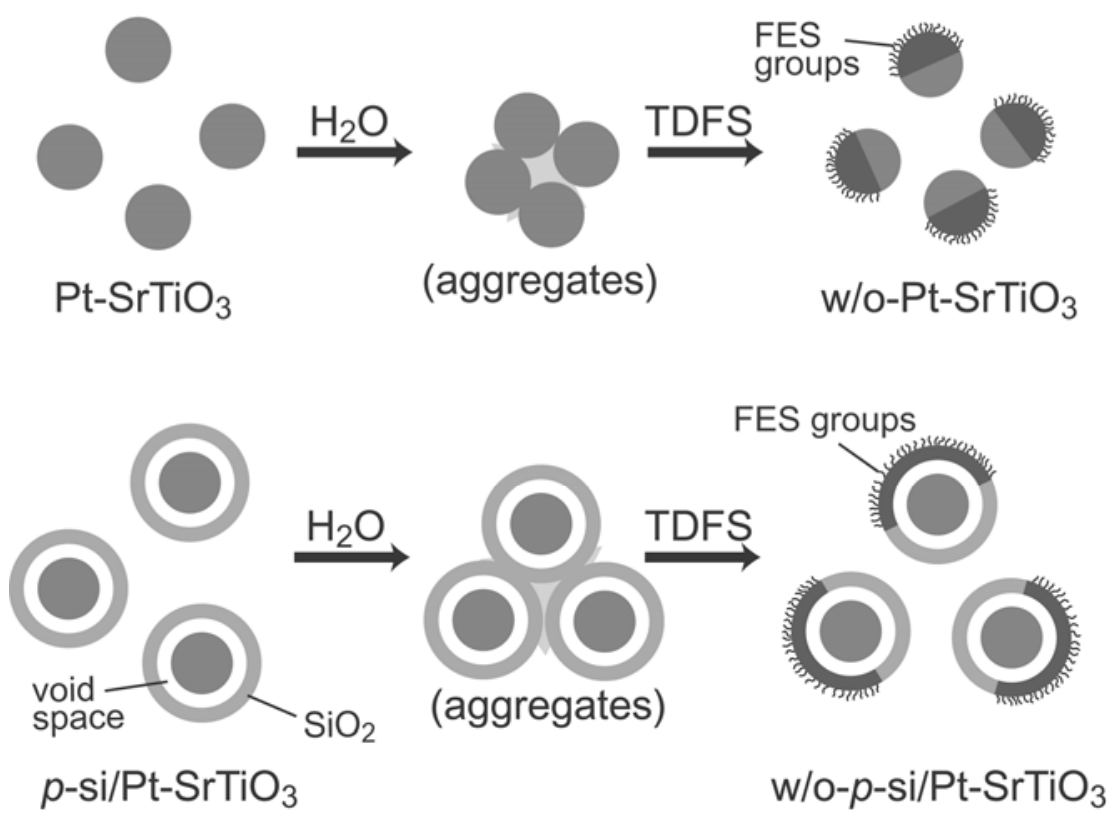

Scheme 1 


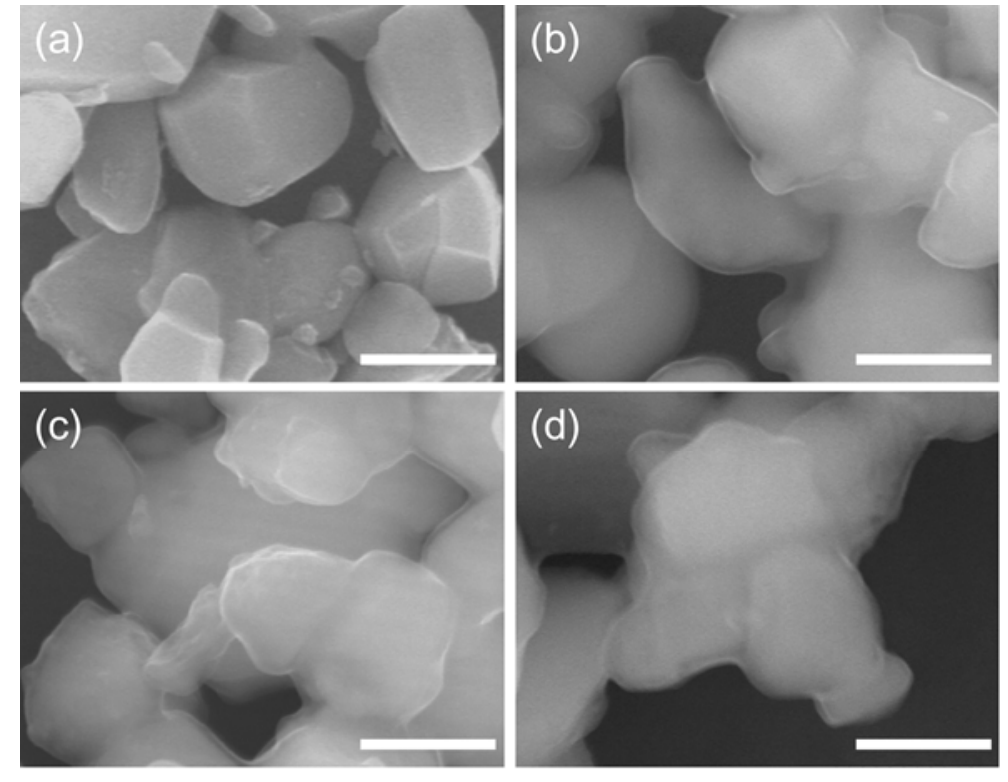

Figure 1 


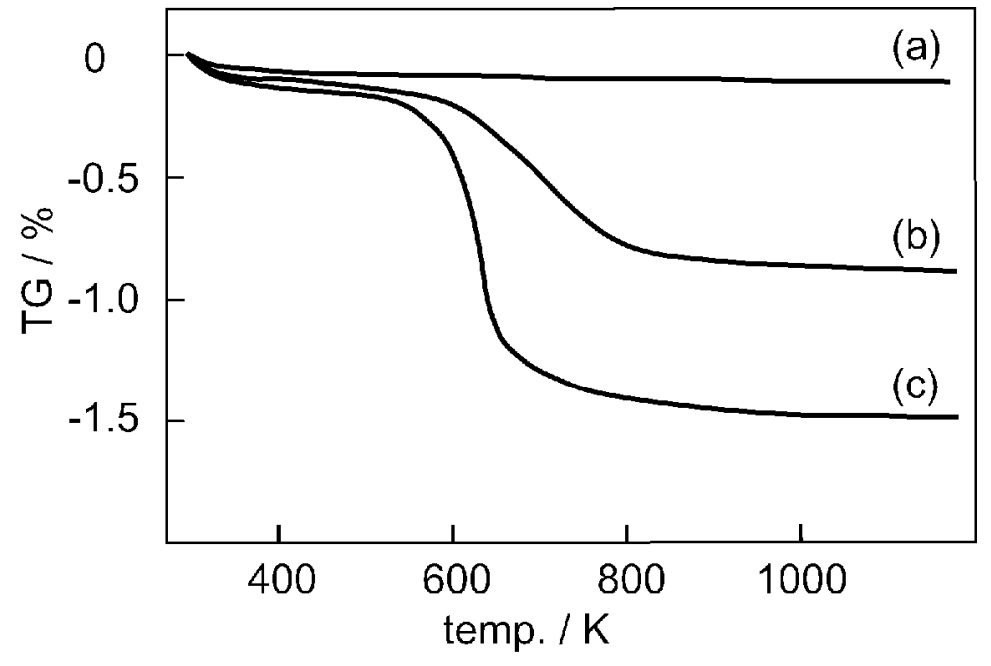

Figure 2 


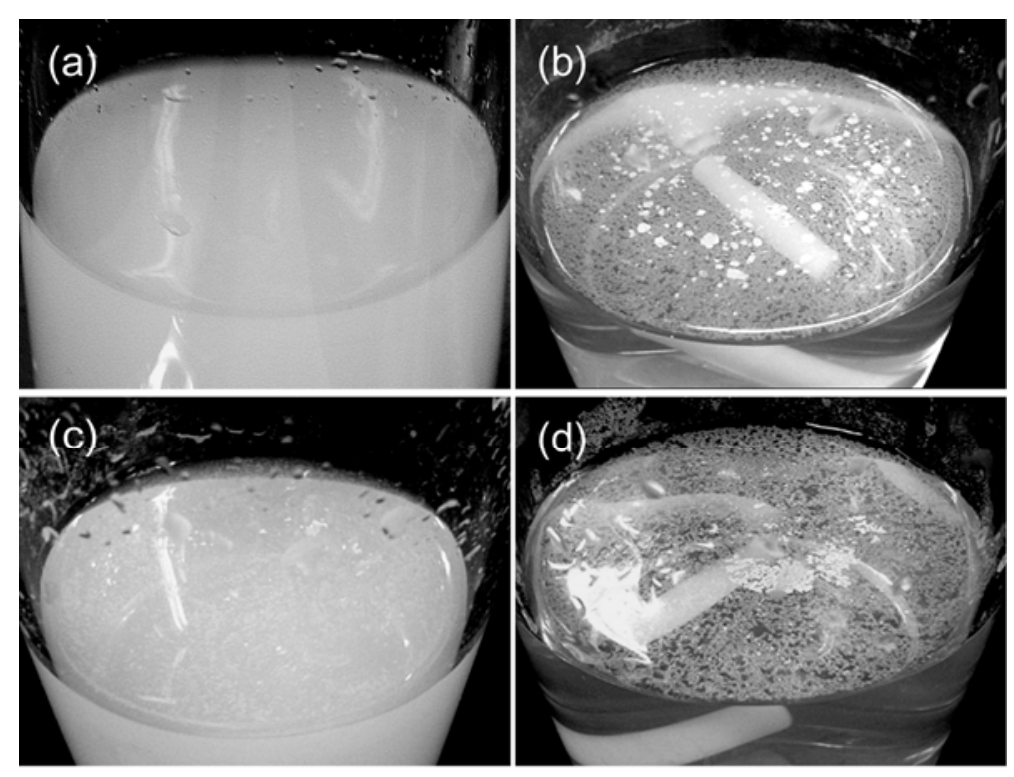

Figure 3 


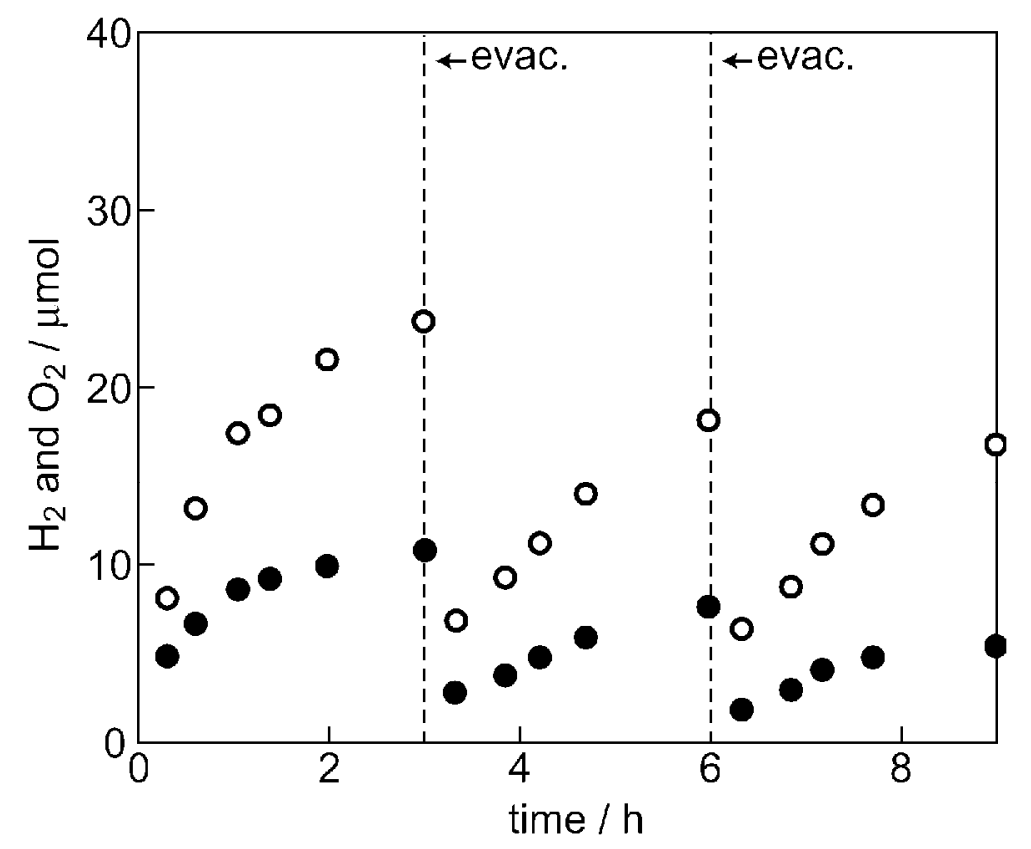

Figure 4 


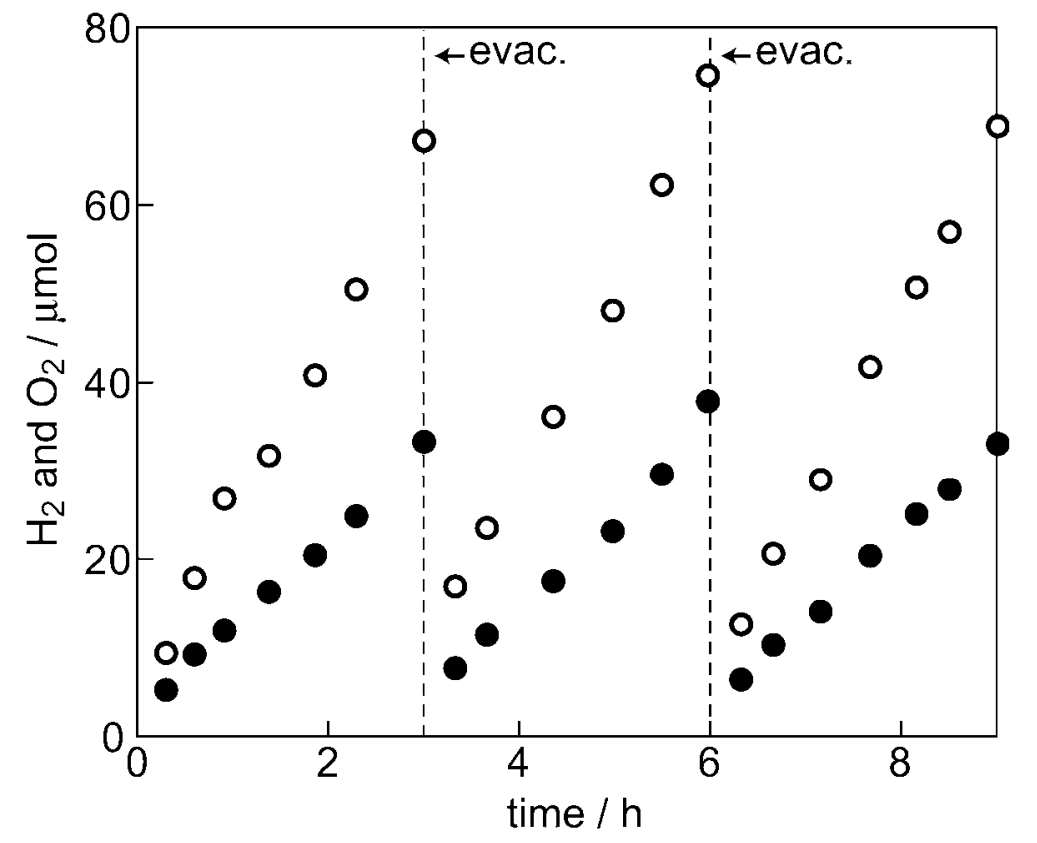

Figure 5 